\title{
ARTICLE
}

\section{CBR antimicrobials alter coupling between the bridge helix and the $\beta$ subunit in RNA polymerase}

Anssi M. Malinen ${ }^{1}$, Monali NandyMazumdar ${ }^{2}$, Matti Turtola ${ }^{1}$, Henri Malmi ${ }^{1}$, Thadee Grocholski ${ }^{1}$, Irina Artsimovitch ${ }^{2} \&$ Georgiy A. Belogurov ${ }^{1}$

Bacterial RNA polymerase (RNAP) is a validated target for antibacterial drugs. CBR703 series antimicrobials allosterically inhibit transcription by binding to a conserved $\alpha$ helix ( $\beta^{\prime}$ bridge helix, $\mathrm{BH}$ ) that interconnects the two largest RNAP subunits. Here we show that disruption of the $\mathrm{BH}-\beta$ subunit contacts by amino-acid substitutions invariably results in accelerated catalysis, slowed-down forward translocation and insensitivity to regulatory pauses. CBR703 partially reverses these effects in CBR-resistant RNAPs while inhibiting catalysis and promoting pausing in CBR-sensitive RNAPs. The differential response of variant RNAPs to CBR703 suggests that the inhibitor binds in a cavity walled by the $\mathrm{BH}$, the $\beta^{\prime} \mathrm{F}$-loop and the $\beta$ fork loop. Collectively, our data are consistent with a model in which the $\beta$ subunit fine tunes RNAP elongation activities by altering the $\mathrm{BH}$ conformation, whereas CBRs deregulate transcription by increasing coupling between the $\mathrm{BH}$ and the $\beta$ subunit.

\footnotetext{
${ }^{1}$ Department of Biochemistry, University of Turku, Turku 20014, Finland. ${ }^{2}$ Department of Microbiology, The Ohio State University, Columbus, Ohio 43210 , USA. Correspondence and requests for materials should be addressed to G.A.B. (email: gebelo@utu.fi)
} 
$\mathrm{R}$ NA polymerase (RNAP) mediates synthesis of an RNA copy of the template DNA-the first and often decisive step in gene expression. All RNAPs transcribing cellular genomes are multisubunit enzymes that share homologous catalytic cores ${ }^{1,2}$. Bacterial RNAP, a five-subunit complex $\alpha \alpha \beta \beta^{\prime} \omega$, is the simplest model system for studies of fundamental mechanistic properties of all multisubunit RNAPs and a validated target for antibacterial drugs ${ }^{3}$.

The cycle of nucleotide incorporation by RNAP is governed by alternate closure and opening of the active site by a $\beta^{\prime}$ subunit mobile domain called trigger loop (TL): catalysis of phosphodiester bond formation involves an obligatory closure ${ }^{4,5}$, whereas translocation along the DNA requires opening of the active site ${ }^{6}$. RNAP also reversibly isomerizes into an off-pathway state that is inhibitory for nucleotide addition. The off-pathway state, aka an elemental pause ${ }^{7}$, is the precursor to all regulatory events during transcription elongation, such as longer-lived pauses and termination $^{8-10}$. Long-lived pauses in turn function to synchronize transcription and translation in prokaryotes ${ }^{11,12}$ and to recruit regulatory proteins to transcribing RNAP in all domains of life ${ }^{13-15}$.

The structural rearrangements accompanying catalysis are relatively well understood. The TL folding into a closed conformation is dependent on the formation of a triple-helical bundle with the $\beta^{\prime}$ subunit bridge helix $(\mathrm{BH})$, a long metastable $\alpha$ helix that spans the active site cleft and moulds into a groove in the $\beta$ subunit $^{4}$ (Fig. 1a). The stability of folded TL is also modulated by its interaction with the $\beta^{\prime}$ F-loop, an aminoterminal extension of $\mathrm{BH}^{16}$. The structural rearrangements associated with isomerization into the elemental pause remain elusive owing to the transient nature of the state. It has been suggested that this isomerization involves fraying of the RNA $3^{\prime}$-end in the active site, kinking of the $\mathrm{BH}$, opening of the $\beta^{\prime}$ clamp domain and changes in the template DNA conformation in the vicinity of the active site $e^{8,17-20}$.

RNAP active site structure evolved to achieve optimal balance between catalytic efficiency, processivity and amenability to regulation $^{21}$. First, the stability of folded TL is tuned up to permit both efficient catalysis and rapid translocation that require folding and unfolding of TL, respectively. Second, the propensity to isomerize into the elemental paused state is tuned up to permit both efficient RNA chain elongation and the proper response to regulatory signals. In this work, we present evidence that conformational coupling between the $\beta$ subunit and $\mathrm{BH}$ plays an important role during elongation by RNAP. We also report plausible structural models of CBR703 ( $N$-hydroxy- $N^{\prime}$-phenyl-3trifluoromethyl-benzamidine) series inhibitors ${ }^{22,23}$ bound in an occluded pocket at the $\mathrm{BH}-\beta$ subunit interface and elucidate mechanistic details of their antibacterial action.

\section{Results}

RNAPs with amino-acid substitutions at BH- $\beta$ interface. To gain insights into the mechanism of action of CBR-type antibiotics, we performed the detailed analysis of elongation activities of five RNAPs with substitutions at the $B H-\beta$ subunit interface, the anticipated binding site of CBRs (Fig. 1b). The BH $\beta^{\prime}$ F773V and F-loop $\beta^{\prime}$ P750L were first identified in a genetic screen for alleles resistant to CBR-type inhibitors ${ }^{22}$. $\beta^{\prime}$ F773V RNAP was later characterized as pause and terminator resistant ${ }^{24}$ and has been suggested to have altered translocation and fidelity ${ }^{25}$. The D-loop $\beta$ P560S,T563I (RpoB5101) RNAP was identified in an in vivo screen as an enzyme with decreased termination ${ }^{26}$. The Fork loop $\beta$ V550A RNAP was designed to probe interactions of $\beta^{\prime} \mathrm{F} 773$ with the $\beta$ Fork loop. The BH $\beta^{\prime}$ H777A RNAP was reported to have relatively unaltered in vitro activity and mild in vivo growth defects in a study by Jovanovic et al. ${ }^{27}$ Here we reevaluated $\beta^{\prime} \mathrm{H} 777 \mathrm{~A}$ properties to probe the clash between CBR703 and one of the $\beta^{\prime} \mathrm{H} 777$ conformers revealed by docking experiments (see below).

Most of experiments in this study were performed with wild type and $\beta^{\prime}$ F773V RNAPs that also contained a BH $\beta^{\prime}$ N792D substitution. The $\beta^{\prime} \mathrm{N} 792 \mathrm{D}$ substitution increased Escherichia coli RNAP sensitivity to streptolydigin (STL) ${ }^{28}$ but did not detectably affect the nucleotide addition and translocation rates as well as translocation bias (this work), enabling us to use low concentrations of STL, which do not interfere with fluorescence measurements, to bias RNAP forward in translocation studies.

We assembled variant RNAP transcription elongation complexes (TECs) on chemically synthesized nucleic-acid scaffolds containing fluorescent 6-methyl-isoxanthopterin (6-MI) base in the template strand and used direct time-resolved translocation ${ }^{6}$ and nucleotide-addition ${ }^{29,30}$ assays to study the effects of substitutions on RNAP translocation equilibrium, translocation rates, catalytic activity and response to CBR703. We also

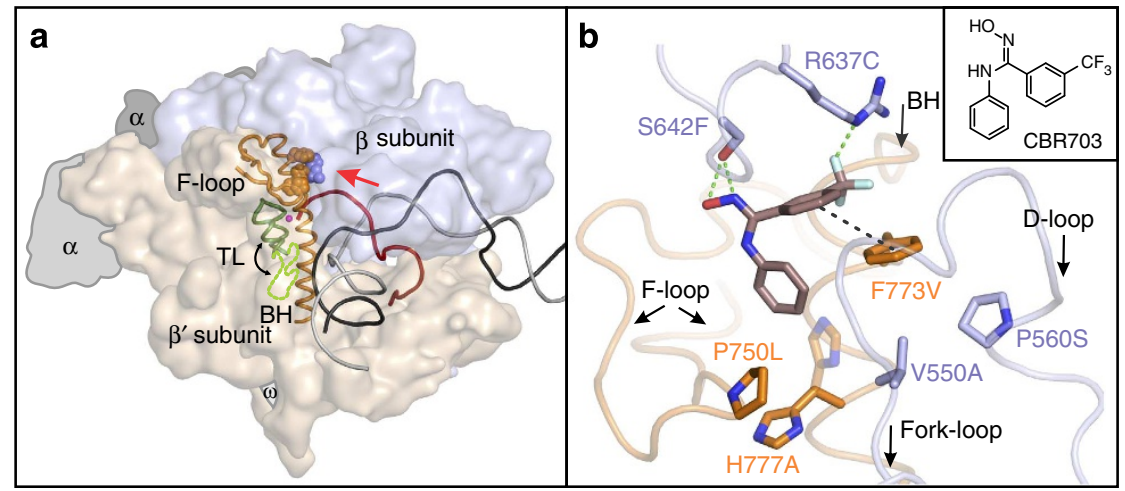

Figure 1 | Binding site of CBR series inhibitors at the BH- $\beta$ subunit interface. (a) An overview of the bacterial TEC. $\beta$ (light blue) and $\beta^{\prime}$ (wheat) are depicted as semi-transparent surfaces, $\alpha$ and $\omega$ (largely obstructed by $\beta^{\prime}$ ) subunits are depicted as flat grey outlines. BH (orange), F-loop (orange), TL (green-closed conformation, dashed light green-open conformation), RNA (red), template (black) and non-template (grey) DNA strands are depicted as cartoons. Amino-acid residues altered in this study are depicted as spheres. A red arrow indicates the direction of the view in $\mathbf{b}$. (b) $C B R 703$ (sticks with brown carbons and inset) docked at the BH- $\beta$ subunit interface of $E$. coli RNAP (PDB 4IGC) ${ }^{37}$. The native amino-acid residues replaced by CBR703resistant and -sensitive ( $\beta$ P560S and $\beta^{\prime} \mathrm{H} 777 \mathrm{~A}$ ) substitutions are depicted as sticks. Cartoons and side chain's carbons of $\beta$ and $\beta^{\prime}$ are coloured pastel blue and orange, respectively. The outwards $s^{37,50,51}$ (opaque) and inwards ${ }^{52}$ (semi-transparent) facing conformers of $E$. coli $\beta^{\prime}$ His 777 are shown. Green and black-dashed lines depict polar and $\pi$-stacking interactions, respectively. Figure was prepared using PyMOL Molecular Graphics System, Version 1.6.0.0; Schrödinger, LLC. The sources of atomic coordinates are listed in Supplementary Table 3. 
compared effects of these substitutions on RNAP response to a regulatory pause site in the presence and absence of CBR703.

$\beta^{\prime}$ F773V and $\beta^{\prime}$ P750L backward bias translocation equilibrium. All five variant RNAPs translocated forward following the incorporation of the cognate GMP, as judged by increase in 6-MI fluorescence. To assess the completeness of translocation, we compared fluorescence intensities of TECs extended by rNMP and $2^{\prime} \mathrm{dNMP}$. The $2^{\prime} \mathrm{dNMP}$-extended TECs are anticipated to display higher level of fluorescence than the rNMP-extended TECs unless the latter are fully post-translocated because $2^{\prime} \mathrm{OH}$ group is essential for stabilizing the pre-translocated state ${ }^{6}$. The $2^{\prime}$ dNMP-extended $\beta^{\prime}$ F773V and $\beta^{\prime}$ P750L TECs displayed brighter fluorescence than the rNMP-extended TECs (Fig. 2a and Supplementary Fig. 1). In contrast, the fluorescence levels of GMP- and $2^{\prime}$ dGMP-extended TECs formed by other RNAPs were the same. Forward-biasing $3^{\prime} \mathrm{dNMP}$ - and rNMP-extended $\beta^{\prime}$ F773V and $\beta^{\prime}$ P750L TECs with the next incoming substrate NTPs and their non-hydrolyzable analogues, respectively, reported fluorescence levels similar to those of the $2^{\prime}$ dNMPextended TECs (Fig. 2a and Supplementary Fig. 1). We concluded that the $2^{\prime} \mathrm{dNMP}$-extended $\beta^{\prime} \mathrm{F} 773 \mathrm{~V}$ and $\beta^{\prime}$ P750L TECs are nearly $100 \%$ post-translocated, whereas AMP- or GMP-extended TECs contain $\sim 40 \%$ and CMP- or UMP-extended TECs contain $\sim 70 \%$ of pre-translocated state. Overall, the above experiments revealed that $\beta^{\prime}$ F773V and $\beta^{\prime}$ P750L RNAPs displayed a measurable fraction of pre-translocated states, whereas other RNAPs in our set were nearly $100 \%$ post-translocated.

$\boldsymbol{\beta}^{\prime}$ F773V RNAP translocation is controlled by TL opening. We employed two antibiotics with established modes of action, tagetitoxin (TGT) and STL, to demonstrate that translocation of $\beta^{\prime}$ F773V TEC is controlled by opening and closure of the active site by the TL. TGT is a high-affinity pyrophosphate analogue that backward biases RNAP by stabilizing the closed active site $^{6,31}$. Indeed, addition of saturating amounts of TGT to rNMP-extended TECs reduced their fluorescence to the level of non-extended TECs, which corresponds to the pre-translocated state (Fig. 3). STL binds to the inner face of the $\mathrm{BH}$ and stabilizes the open active site conformation, favouring the post-translocated state ${ }^{28,32}$. Indeed, addition of saturating amounts of STL to rNMP-extended TECs increased their fluorescence to the level of $2^{\prime}$ dNMP-extended TECs, which correspond to the post-translocated state (Fig. 2b). CBR703 also forward biased $\beta^{\prime}$ F773V TECs in a concentration-dependent manner, although it was less potent than STL and failed to quantitatively move the TECs into the post-translocated state (Fig. 2b). Both STL and CBR703 forward biased wild-type RNAP, as evident from their ability to offset the effect of TGT on translocation equilibrium (Fig. 2c). The response of $\beta^{\prime}$ F773V RNAP to STL suggests that the shift of translocation equilibrium towards the pre-translocated state in this RNAP originates, at least in part, from an increased stability of the folded TL. The ability of CBR703 to forward bias $\beta^{\prime}$ F773V and wild-type RNAPs suggests that the inhibitor destabilizes the folded TL.

Altered RNAPs have decreased forward translocation rate. We performed parallel time-resolved measurements of nucleotide addition and translocation for GMP (and CMP in case of $\beta^{\prime}$ F773V RNAP) addition reactions. The forward and backward translocation rates were inferred from a delay between nucleotide addition and translocation curves using a reversible translocation model, as described in Supplementary Methods. These analyses revealed that RpoB5101 and $\beta$ V550A substitutions reduced the forward translocation rate to $30-40 \mathrm{~s}^{-1}$ (from $60 \mathrm{~s}^{-1}$ in wildtype RNAP), whereas $\beta^{\prime} \mathrm{F} 773 \mathrm{~V}$ and $\beta^{\prime} \mathrm{P} 750 \mathrm{~L}$ substitutions reduced the rate to $9 \mathrm{~s}^{-1}$ (Fig. 3 and Supplementary Fig. 2). The backward translocation rate was estimated at $6 \mathrm{~s}^{-1}$ for GMP-extended $\beta^{\prime}$ F773V and $\beta^{\prime}$ P750L TECs and at $20 \mathrm{~s}^{-1}$ for CMP-extended $\beta^{\prime}$ F773V TEC (Fig. 3d), but did not measurably contribute to the kinetics of fluorescence change in other RNAPs and was fixed to zero during analyses. Whereas the $6 \mathrm{~s}^{-1}$ rate could be potentially masked by $60 \mathrm{~s}^{-1}$ forward translocation rate in wild-type RNAP (assuming 10\% uncertainly in determination
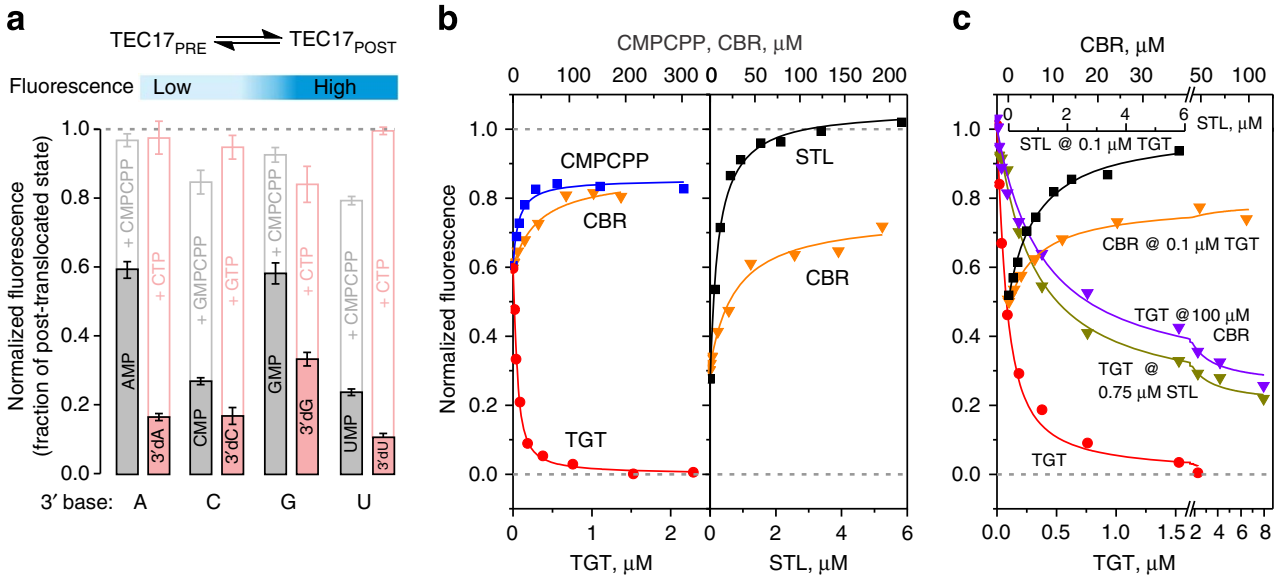

Figure 2 | Effects of CBR703 and substitutions at the BH- $\boldsymbol{\beta}$ subunit interface on translocation equilibrium. Top schematic describes the experimental set-up. Best fit curves were simulated using parameters described in Supplementary Table 4. Fluorescence data were averaged over two to three experiments. (a) $\beta^{\prime}$ F773V TECs display measurable fractions of the pre-translocated state. Fluorescence of rNMP (grey fill) and $3^{\prime} \mathrm{dNMP}$ (pink fill)extended TECs normalized to the level of $2^{\prime}$ dNMP-extended TECs. White bars depict the effects of the next substrate NTP (pink outline) or its nonhydrolyzable analogue (grey outline). Error bars are s.d. (b) CBR703 and STL forward-biased $\beta^{\prime}$ F773V RNAP sensitized to STL by the $\beta^{\prime}$ N792D substitution. Left panel: GMP-extended TEC. Right panel: CMP-extended TEC. TGT (red) and STL (black) quantitatively move the TECs into pre- and post-translocated states, respectively. CBR703 (orange) and cytidine- $5^{\prime}-[(\alpha, \beta)$-methyleno]triphosphate (CMPCPP; blue) measurably forward bias the TECs. (c) STL and CBR703 offset TGT effects on wild-type RNAP sensitized to STL by $\beta^{\prime}$ N792D substitution. TGT (red) quantitatively converts post-translocated GMP-extended TEC into pre-translocated TEC. TGT is less potent in backward-biasing RNAP in the presence of $0.75 \mu \mathrm{M} \mathrm{STL}$ (olive) and $100 \mu \mathrm{M}$ CBR703 (purple). STL (black) and CBR703 (orange) forward bias RNAP in the presence of $0.1 \mu \mathrm{M}$ TGT. 


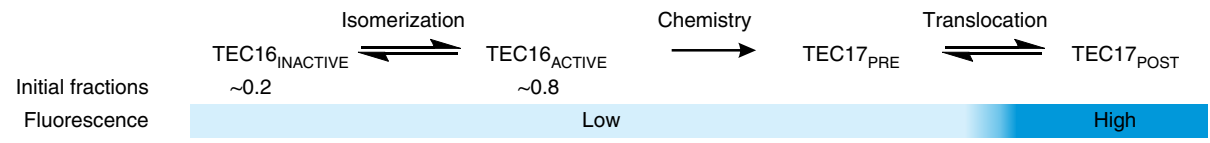

a

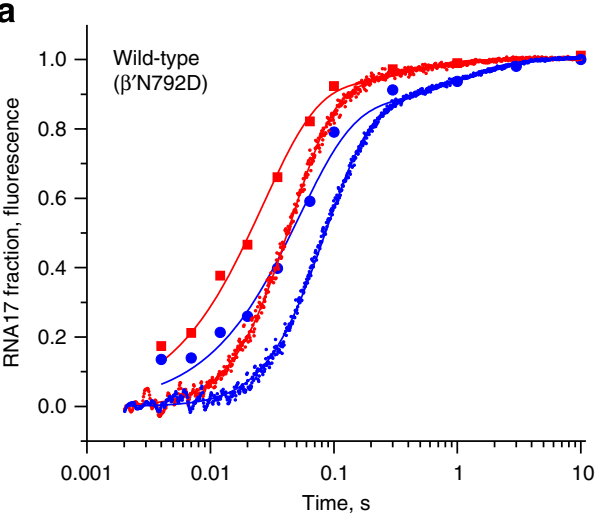

C
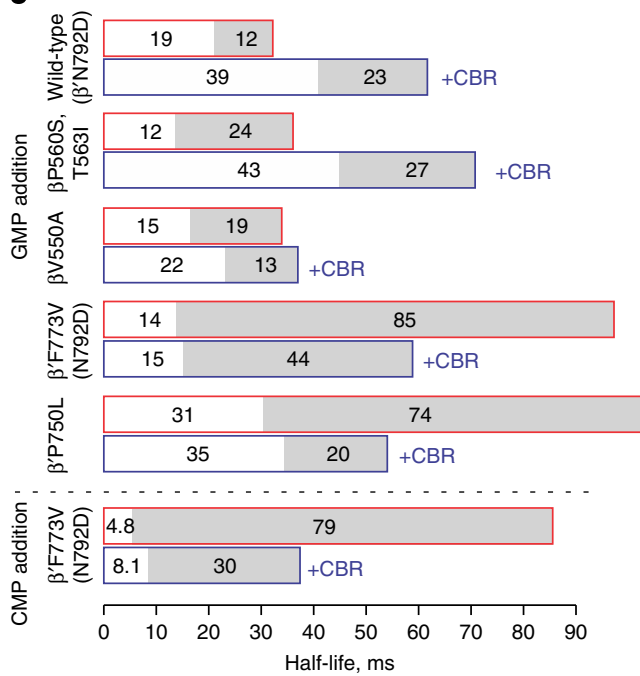

Nucleotide addition Forward translocation

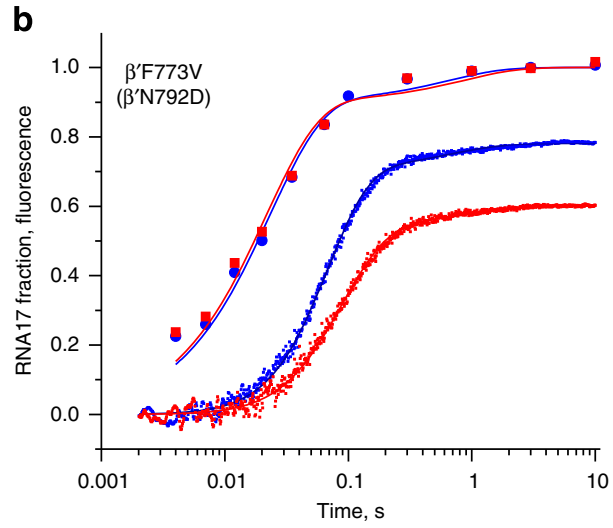

d

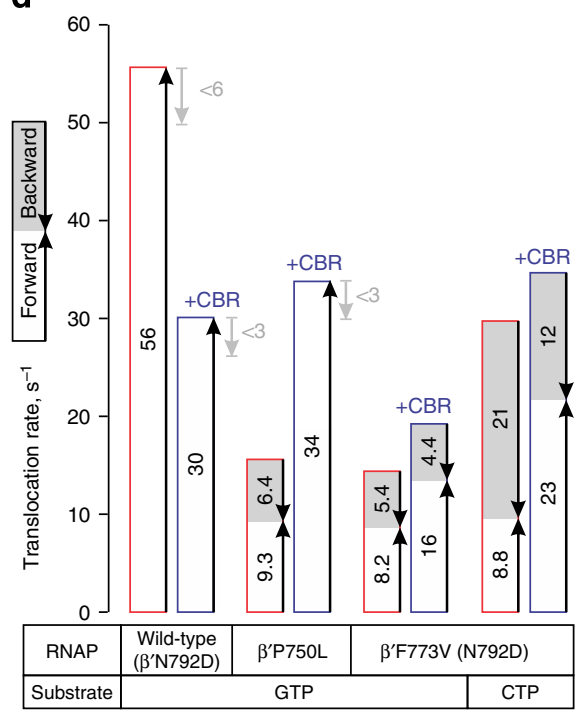

Figure 3 | Effects of CBR703 and substitutions at the BH- $\beta$ subunit interface on nucleotide addition and translocation rates. Top schematic describes the experimental set-up. CBR703 is present at $100 \mu \mathrm{M}$ where indicated (blue outline). Best fit curves and bar graphs were drawn using parameters described in Supplementary Table 5. (a,b) Time courses of GMP addition (discrete time points) and translocation (continuous trace) by wild-type (a) and $\beta^{\prime}$ F773V (b) RNAPs. Nucleotide addition and translocation assays were performed in duplicate; translocation curves are averages from six to nine time traces. (c) Half-lives of nucleotide addition cycles calculated as sums of nucleotide addition (white fill) and forward translocation (grey fill) half-lives. (d) The apparent translocation rates of wild-type ( $\beta^{\prime}$ N792D), $\beta^{\prime}$ P750L and $\beta^{\prime}$ F773V, $\beta^{\prime}$ N792D RNAPs depicted as sums of forward (white fill) and backward (grey fill) translocation rates. The representation reflects the relationship between the above three rates defined by formal kinetic rules for a reversible reaction. For wild-type RNAP and $\beta^{\prime} \mathrm{P} 750 \mathrm{~L}$ (in the presence of CBR703), the backward rates are constrained to zero during the fit but may potentially constitute up to $10 \%$ of forward rate (grey arrows) assuming 10\% uncertainty in determination of fluorescent levels of extended TECs.

of equilibrium levels of fluorescence), the $20 \mathrm{~s}^{-1}$ value strongly suggests that $\beta^{\prime} \mathrm{F} 773 \mathrm{~V}$ substitution increases backward translocation rate. The shift of translocation equilibrium towards the pre-translocated state in $\beta^{\prime} F 773 \mathrm{~V}$ RNAP thus originates from both the decrease in forward and increase in backward translocation rates. The decrease in $\mathrm{BH}$ mobility or bendability ${ }^{25}$ alone is not sufficient to explain such observations, whereas stabilization of the folded $\mathrm{TL}$, in part as a consequence of less bendable $\mathrm{BH}$, satisfactory explains the observed effects.

Altered RNAPs are differentially sensitive to CBR703. We investigated the effect of CBR703 on nucleotide addition and translocation rates of variant RNAPs in the single nucleotide addition assay. Preincubation of TECs with $100 \mu \mathrm{M}$ CBR703 lengthened nucleotide addition cycle twofold in the wild-type and RpoB5101 RNAPs, had little effect on $\beta$ V550A and facilitated the completion of the cycle by $\beta^{\prime} \mathrm{P} 750 \mathrm{~L}$ and $\beta^{\prime}$ F773V RNAPs twofold (Fig. 3c). $\beta^{\prime}$ H777A RNAP was inhibited nearly 250 -fold and is discussed in a separate section (see below). At the level of individual steps, nucleotide addition was unaffected or inhibited, whereas forward translocation was marginally inhibited in wildtype RNAP, unaffected in RpoB5101 and stimulated in $\beta V 550 \mathrm{~A}$, $\beta^{\prime}$ P750L and $\beta^{\prime}$ F773V RNAPs (Fig. 3c). CBR703 also reduced backward translocation rate of $\beta^{\prime}$ P750L TEC below the detection threshold and marginally slowed-down backward translocation of $\beta^{\prime}$ F773V TECs (Fig. 3d). The effect of CBR703 on translocation rates of $\beta^{\prime}$ P750L and $\beta^{\prime}$ F773V RNAPs is consistent with its 
forward-biasing effect in the equilibrium assay (Fig. 2b) and reinforces the hypothesis that inhibitor promotes the TL unfolding. Consistently, with the results of single nucleotide addition assays, CBR703 permitted rapid processive transcription through the long template by $\beta^{\prime} \mathrm{F} 773 \mathrm{~V}, \beta^{\prime} \mathrm{P} 750 \mathrm{~L}$ and $\beta \mathrm{V} 550 \mathrm{~A}$ RNAPs (Fig. 4 and Supplementary Fig. 3). In contrast, transcription by wild-type RNAP was significantly impeded by multiple pauses.

Altered RNAPs have reduced sensitivity to regulatory pause. $\beta^{\prime}$ F773V and RpoB5101 RNAPs are among the least pause responsive $E$. coli RNAPs hitherto characterized ${ }^{24}$. To find out whether pause insensitivity is a general property of RNAPs with substitutions at the $\mathrm{BH}-\beta$ subunit interface, we evaluated their response to the hairpin-stabilized his $P$ pause element using a standard single-round in vitro transcription assay ${ }^{33}$. We used the pIA171 linear transcription template on which the his $P$ was positioned downstream from a strong T7A1 promoter (Fig. 4). On this template, radiolabelled transcription complexes can be halted at position A29 when transcription is initiated in the absence of UTP, with ApU dinucleotide, ATP, GTP and $\alpha-\left[{ }^{32} \mathrm{P}\right]$ CTP. The halted A29 complexes can then be chased on addition of all four NTP substrates. We found that, similar to $\beta^{\prime} \mathrm{F} 773 \mathrm{~V}$ and RpoB5101 RNAPs characterized earlier ${ }^{24}, \beta^{\prime} \mathrm{P} 750 \mathrm{~L}$ and $\beta$ V550A RNAPs were relatively resistant to the hisP pause
(Fig. 4 and Supplementary Fig. 3), suggesting that weakening $\mathrm{BH}-\beta$ subunit contacts universally leads to insensitivity to regulatory pauses. Strikingly, the sensitivity of $\beta^{\prime} \mathrm{F} 773 \mathrm{~V}$, $\beta^{\prime} \mathrm{P} 750 \mathrm{~L}$ and $\beta \mathrm{V} 550 \mathrm{~A}$ RNAPs to hisP pause was partially restored in the presence of CBR703. The latter result suggests that CBR703 promotes formation of native intermediates in the pausing pathway.

In silico docking of CBRs at the BH- $\beta$ interface. It has been anticipated for some time that CBR-type compounds bind at the $\mathrm{BH}-\beta$ subunit interface of $E$. coli RNAP near $\beta$ 'F773 based on extensive set of in vivo selected CBR703- and CBR9379-resistant mutations $^{22}$. Here we used AutoDock Vina ${ }^{34}$ and GOLD ${ }^{35,36}$ programs, which rely on different algorithms and scoring functions, to identify the binding sites for CBR703, CBR9379 and CBR9393 in E. coli RNAP holoenzyme crystal structure (PDB 4IGC) ${ }^{37}$. Both Vina and GOLD failed to identify a common binding mode for the three inhibitors to rigid RNAP but robustly recovered overlapping binding sites for CBR9379 and CBR9393 when $\beta^{\prime}$ Leu770 and $\beta^{\prime}$ Phe773 side chains were set flexible (Supplementary Fig. 4). Moreover, Vina independently recovered the same binding mode for CBR703 (Fig. 1b), a relatively symmetric substructure of CBR9379.

In the resulting models, the structural moieties common for the three CBRs are positioned in a spacious cavity walled by the $\mathrm{BH}$,

a
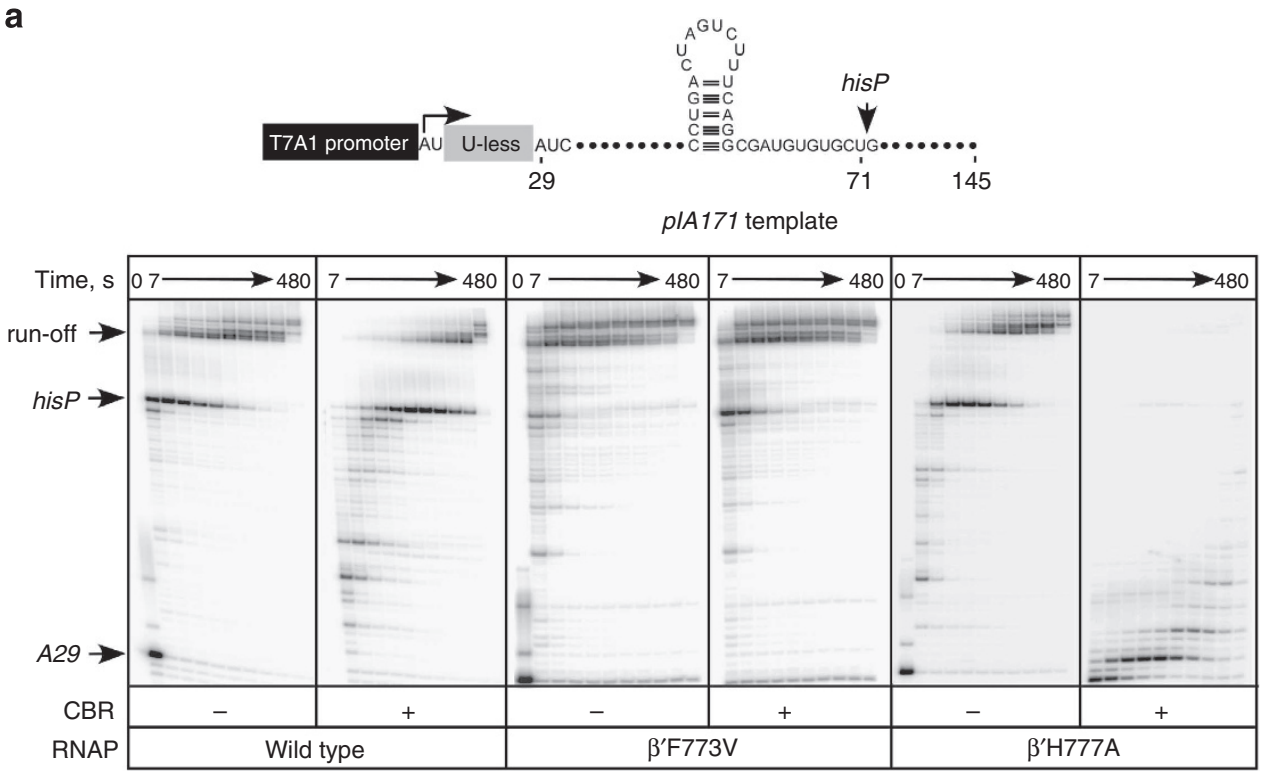

b

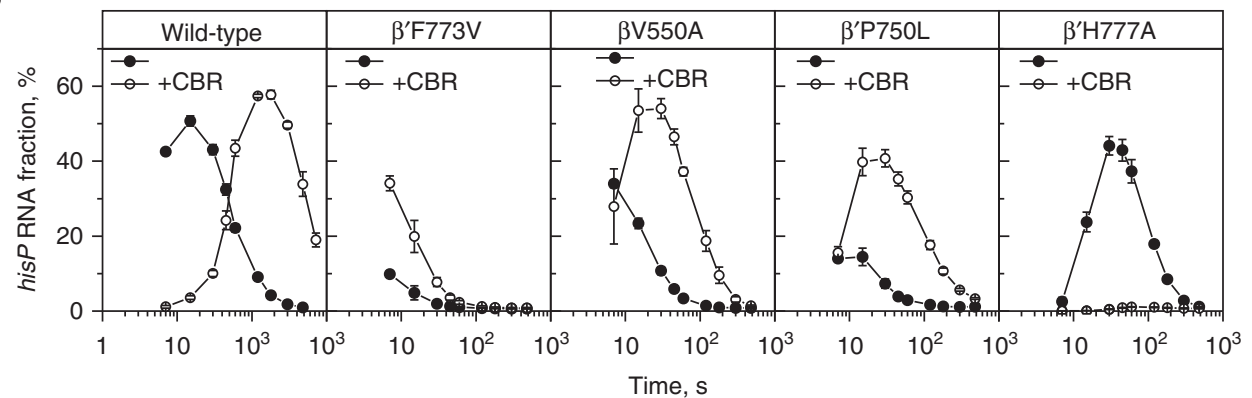

Figure 4 | Effects of CBR703 and substitutions at the BH- $\beta$ subunit interface on pausing at the regulatory pause site. (a) Transcript elongation on pIA171 template by wild-type, $\beta^{\prime}$ F773V and $\beta^{\prime}$ H777A RNAPs in the absence (left panels) or in the presence (right panels) of $100 \mu M$ CBR703.

(b) Quantification of the fraction of RNA at the hisP (transcript position U71) from the data shown in a and Supplementary Fig. 3. Fraction refers to the ratio of the paused RNA to the sum of all products resulting from elongation of TEC halted at A29. Error bars are s.d. of three independent experiments. 


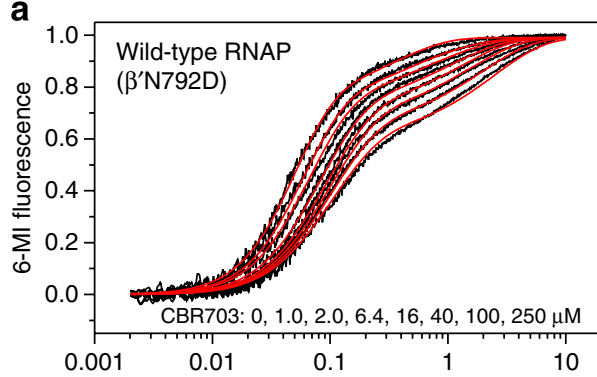

b

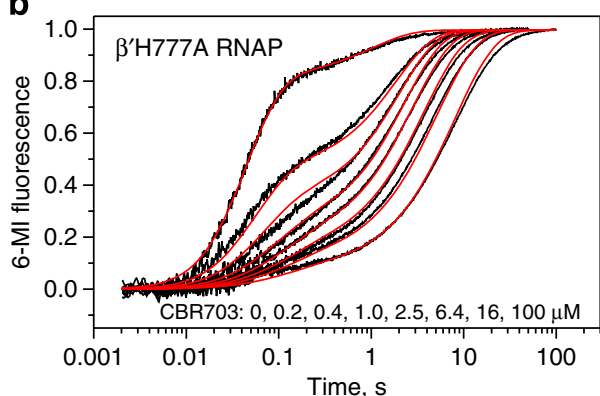

C



Figure 5 | CBR703 slows down catalysis and promotes isomerization into the inactive state. Wild-type (a) and $\beta^{\prime} \mathrm{H} 777 \mathrm{~A}(\mathbf{b})$ TEC16s are preincubated with different concentrations of CBR703, rapidly mixed with GTP and formation of post-translocated TEC17s is monitored by continuous recording of 6-Ml fluorescence (black traces). Best fit curves (red) were simulated using parameters described in c and Supplementary Methods. The experiments were performed in duplicate, fluorescence curves are averages from three to seven time traces. (c) Kinetic scheme describing the effects of CBR703 on completion of GMP addition cycle in $\mathbf{a}$ and $\mathbf{b}$. Grey boxes and red font accentuate parameters affected by CBR703 and $\beta^{\prime} \mathrm{H} 777 \mathrm{~A}$ substitution, respectively.

Fork loop and F-loop and interact with key residues implicated in resistance to CBRs (Fig. $1 \mathrm{~b}$ and Supplementary Fig. 4) Trifluoromethyl group forms hydrogen bond with $\beta$ Arg637 and interacts with aromatic $\pi$-system of $\beta^{\prime}$ Phe773. Trifluoromethylated benzene ring stacks with $\beta^{\prime}$ Phe773 in an offset parallel configuration, whereas the second benzene ring contacts $\beta^{\prime}$ Pro750, $\beta^{\prime}$ Ile774 and $\beta$ Val550. The $N$-hydroxyamidine moieties interconnecting benzene rings in CBR703 and CBR9379 interact with hydroxyl group and main chain carbonyl of $\beta$ Ser642. Bulky substituents in position 4 (in CBR9393) and 5 (in CBR9379) of trifluoromethylated ring extend into $\beta$ lobe, form multiple van der Waals interactions with RNAP side chains and hydrogen bond with $\beta$ Glu611 (in CBR9393-RNAP complex) and $\beta$ Pro552 main chain carbonyl (in CBR9379-RNAP complex) accounting for higher potency of the larger CBRs (Supplementary Fig. 4). The atomic coordinates of CBR703, CBR9379 and CBR9393-RNAP complexes are provided as Supplementary Data $1-3$, respectively.

We noted that in Thermus thermophilus RNAP structures, the orientation of the $\mathrm{BH} \beta^{\prime} \mathrm{His} 1075$ side chain is different from that of the homologous $\beta^{\prime}$ His777 in the crystal structure of $E$. coli RNAP holoenzyme that we used for docking experiments. The $\beta$ 'His 1075 side chain extends into the CBR703-binding cavity and clashes with the unsubstituted aromatic ring of CBR703 (Fig. 1b). This observation suggests that the E. coli $\beta$ 'His777 side chain may alternate between the inwards and outwards facing conformations, interfering with CBRs binding in the former state. In support of the docking model, the $\beta^{\prime} \mathrm{H} 777 \mathrm{~A}$ substitution increased E. coli RNAP affinity for CBR703 fivefold (Fig. 5).

CBR703 promotes TEC isomerization into an inactive state. All assembled TECs that we characterized to date contain $5-25 \%$ of a slow reacting TEC $\left(1-2 s^{-1}\right)^{6}$. The slow fraction originates at or before the nucleotide addition step, but is only well resolved in translocation traces because of dense temporal sampling. We noted that CBR703 increased the fraction of slow TEC in CBRsensitive (wild type and RpoB5101) but not CBR-resistant $\left(\beta V 550 A\right.$ and $\beta^{\prime}$ F773V) RNAPs (Fig. 3 and Supplementary Fig. 2). The increase in fraction of slow TEC is particularly apparent in a set of translocation time curves of the wild-type RNAP recorded at increasing concentration of CBR703 (Fig. 5a) and becomes explicit when the CBR-hypersensitive $\beta^{\prime} \mathrm{H} 777 \mathrm{~A}$ RNAP is used (Fig. 5b). We found that the simplest kinetic model consistent with the wild-type and $\beta^{\prime} \mathrm{H} 777 \mathrm{~A}$ RNAPs data postulates that the slow TEC is an inactive TEC in slow equilibrium with an active TEC (Fig. 5c). Note that such definition of a slow TEC matches the definition of a paused TEC. The isomerization rate constants were also in the order of those estimated for the elemental pause in single molecule experiments $^{9,38}$. The model predicts that CBR has a dual effect on transcription: it slows down nucleotide addition twofold and promotes isomerization of active TECs into inactive TECs. A stronger effect of CBR703 on $\beta^{\prime} \mathrm{H} 777 \mathrm{~A}$ RNAP originates from changes in two distinct equilibriums: first, the substitution increases CBR703-binding affinity fivefold; second, CBR703 increases bias towards the inactive state 38 -fold in $\beta^{\prime} \mathrm{H} 777 \mathrm{~A}$ RNAP $\left(K_{\text {iso }}=0.26, K_{\text {iso }}^{\mathrm{CBR}}=10\right)$ but has only 2.5 -fold effect in the wild-type RNAP $\left(K_{\text {iso }}=0.35, K_{\text {iso }}^{\mathrm{CBR}}=0.83\right)$. The capacity of CBR703 to promote TEC isomerization into an inactive state inferred from single nucleotide addition experiments in Fig. 5 is entirely consistent with the CBR703 effects on transcription through the long template in Fig. 4 and Supplementary Fig. 3, where CBR703 gradually halts $\beta^{\prime}$ H777A RNAP at multiple sites and restores pause sensitivity of CBR-resistant RNAPs.

\section{Discussion}

Collectively, our data are consistent with the model where disruption of the $\mathrm{BH}-\beta$ subunit contacts relaxes RNAP into a 
ground state characterized by the predominantly closed active site, fast and error-prone catalysis, slow translocation and insensitivity to regulatory pauses. $\beta^{\prime}$ F773V RNAP is a quintessential example of such RNAP. In contrast, in the wild-type RNAP, motions of the $\beta$ subunit modulate on-pathway elongation and isomerization into off-pathway states (Fig. 6). Specifically, conformational coupling between the $\mathrm{BH}$ and $\beta$ subunit destabilizes BH-TL interactions in a controllable manner, thereby fine tuning translocation and catalysis. Perhaps independently, the $\beta$ subunit- $\mathrm{BH}$ interactions also control the equilibrium between the active and paused states of the TEC via the $\mathrm{BH}$ anchor and switch 1 regions ${ }^{19,20,39,40}$.

From a structural perspective, decoupling from the $\beta$ subunit enables the $\mathrm{BH}$ to adopt its native helical conformation and to form multiple interactions with the folded TL, thereby promoting the active site closure. The lack of $\beta$-induced distortions in the $\mathrm{BH}$ structure also diminishes transitions associated with pausing: isomerization of template DNA strand and opening of the clamp domain, which have been structurally linked to the distorted $\mathrm{BH}^{20,41}$. In contrast, binding of CBR inhibitors fills the void at the BH- $\beta$ subunit interface and strengthens $\beta$ subunit interactions with the $\mathrm{BH}$. As a result, the BH-TL interactions are weakened, the folded TL is destabilized and catalysis is slowed down. The $\beta$ subunit also induces larger distortions in the $\mathrm{BH}$ conformation, increasing RNAP propensity to isomerize into inactive state(s) that kinetically resembles the natural intermediates of the pausing pathway. The latter effect may play the major role in the antibacterial action of CBR inhibitors. Whereas the threefold decrease in elongation rate slows down bacterial growth, a small
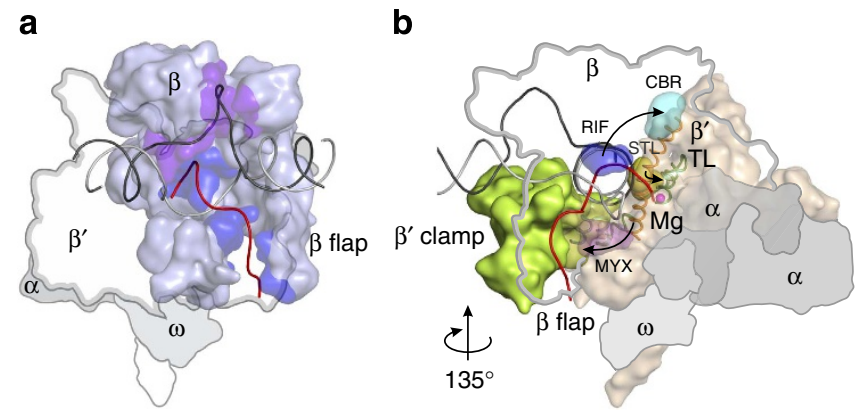

Figure 6 | Antibiotic-binding sites outline an allosteric path mediating regulation of transcription elongation. (a) The $\beta$ subunit (light blue) interacts with nucleic-acid determinants implicated in regulation of transcription elongation ${ }^{53-56}$ : the nascent RNA ${ }^{57}$ (red, blue $\beta$ surface) and unpaired non-template DNA ${ }^{52,58}$ (black, purple $\beta$ surface). $\beta^{\prime}$ subunit is depicted as a contour, and $\alpha$ and $\omega$ subunits as grey outlines. (b) Allosteric effects (black arrows) travel through rifampicin (RIF, sterically ${ }^{59}$ and allosterically ${ }^{60}$ inhibits transcription initiation) -binding site (blue surface, amino-acid substitutions alter RNAP pausing propensities ${ }^{26}$ ) in the $\beta$ subunit. CBR (inhibits transcription elongation by promoting pausing)binding site (cyan surface, amino-acid substitutions lead to pausing defects) outlines the interface where allosteric effects are transferred to the $\mathrm{BH}$ (orange cartoon). STL (inhibits transcription elongation by restricting TL and $\mathrm{BH}$ conformations ${ }^{28,32}$ ) -binding site (yellow surface) marks the region where conformational changes in $\mathrm{BH}$ modulate the stability of the folded TL (green cartoon). Myxopyronin (restricts mobility of the $\beta^{\prime}$ clamp $p^{61-63}$ and alters position of template DNA strand ${ }^{64}$ ) binds to the flexible region (pink surface, switch 1 as green cartoon on the background) that controls movement of $\beta^{\prime}$ clamp (green surface) and conformation of the template DNA strand (grey cartoon). $\beta$ subunit is depicted as a contour, and $\alpha$ and $\omega$ subunits as grey outlines. The figure was prepared using PyMOL Molecular Graphics System, Version 1.6.0.0; Schrödinger, LLC. The sources of atomic coordinates are listed in Supplementary Table 3. increase in propensity to isomerize into the inactive state at each sequence position increases the frequency of long-lived pause and arrest events, ultimately leading to premature cessation of transcription that is detrimental for cell viability. Similarly, we hypothesize that deregulated transcription, rather than the slow forward translocation rate, makes $\beta^{\prime} \mathrm{F} 773 \mathrm{~V}$ and $\beta^{\prime} \mathrm{P} 750 \mathrm{~L}$ RNAPs inviable in the absence of $\mathrm{CBRs}^{22}$. Binding of CBR inhibitors restores the coupling between the $\mathrm{BH}$ and $\beta$ subunit in these RNAPs, thereby restoring transcriptional regulation and supporting viability of the mutant strains.

\section{Methods}

Proteins and reagents. DNA and RNA oligonucleotides were purchased from IBA Biotech (Göttingen, Germany) and Fidelity Systems (Gaithersburg, MD, USA). TGT was from Epicentre (Madison, WI, USA), CBR703 from Maybridge (Tintagel, UK) and STL from Sourcon-Padena (Tübingen, Germany). Cytidine- $5^{\prime}-[(\alpha, \beta)$ methyleno]triphosphate and guanine- $5^{\prime}-[(\alpha, \beta)$-methyleno] triphosphate were from Jena Bioscience (Jena, Germany). RNAPs and yeast inorganic pyrophosphatase were expressed and purified as described previously ${ }^{42,43}$. Plasmids are listed in Supplementary Table 1. Template strand oligonucleotides and RNA primers are listed in Supplementary Table 2. Schematics of all nucleic-acid scaffolds used in this study are shown in Supplementary Fig. 5.

TEC assembly. TECs $(1 \mu \mathrm{M})$ were assembled by a procedure developed by Komissarova et al. ${ }^{44}$ An RNA primer labelled with Atto680 fluorescent dye at the $5^{\prime}$-end was annealed to template DNA, and incubated with $1.5 \mu \mathrm{M}$ RNAP for $10 \mathrm{~min}$ at $25^{\circ} \mathrm{C}$ in TB10D buffer $\left(10 \mathrm{mM} \mathrm{MgCl}_{2}, 40 \mathrm{mM}\right.$ HEPES-KOH pH 7.5, $80 \mathrm{mM} \mathrm{KCl}, 5 \%$ glycerol, $2.5 \%$ dimethylsulphoxide, $0.1 \mathrm{mM}$ EDTA and $0.1 \mathrm{mM}$ DTT) and with $2 \mu \mathrm{M}$ of the non-template DNA for $20 \mathrm{~min}$ at $25^{\circ} \mathrm{C}$. For TECs used in nucleotide addition measurements, RNA was the limiting component at $1 \mu \mathrm{M}$ and the template strand was used at $1.4 \mu \mathrm{M}$, whereas for TECs used in translocation, the template strand was limiting at $1 \mu \mathrm{M}$ and RNA was added at $1.4 \mu \mathrm{M}$.

Nucleotide addition measurements. To determine the incorporation efficiency of NTP, $2^{\prime}$ and $3^{\prime} \mathrm{dNTP}$ substrates, $1 \mu \mathrm{M}$ TEC in $20 \mu \mathrm{l}$ of TB10D buffer was incubated for $10 \mathrm{~min}$ with $5 \mu \mathrm{M}$ substrates at $25^{\circ} \mathrm{C}$ and quenched by adding $80 \mu \mathrm{l}$ of loading buffer ( $94 \%$ formamide, $13 \mathrm{mM} \mathrm{Li}_{4}$-EDTA and $0.2 \%$ Orange G). Time-resolved measurements were performed in an RQF 3 quench-flow instrument (KinTek Corporation, Austin, TX, USA). The reaction was initiated by rapid mixing of $14 \mu \mathrm{l}$ of $0.4 \mu \mathrm{M}$ TEC with $14 \mu \mathrm{l}$ of $400 \mu \mathrm{M}$ NTP. Both TEC and NTP solutions were prepared in TB10D buffer and, where indicated, supplemented with $100 \mu \mathrm{M}$ CBR703. The reaction was allowed to proceed for $0.004-10 \mathrm{~s}$ at $25^{\circ} \mathrm{C}$, quenched with $86 \mu \mathrm{l}$ of $0.5 \mathrm{M} \mathrm{HCl}$ and immediately neutralized by adding $171 \mu \mathrm{l}$ of loading buffer (290 mM Tris base, $13 \mathrm{mM}$ EDTA, 0.2\% Orange G, 94\% formamide). RNAs were separated on $16 \%$ denaturing polyacrylamide gels and visualized with Odyssey Infrared Imager (Li-Cor Biosciences, Lincoln, NE, USA); band intensities were quantified using Image J software ${ }^{45}$.

Translocation measurements. RNAP translocation was assayed by monitoring changes in fluorescence of 6-MI base incorporated into template DNA . Equilibrium levels of fluorescence were determined by recording emission spectra of 6-MI (excitation at $340 \mathrm{~nm}$ ) with an LS-55 spectrofluorometer (Perkin Elmer, Waltham, MA, USA) at $25^{\circ} \mathrm{C}$. The fluorescence at peak emission wavelength $(420 \mathrm{~nm})$ was used for data analysis and representation. Preassembled TECs were diluted at 50-100 nM into $500 \mu \mathrm{l}$ of TB10D buffer, supplemented with $40 \mathrm{pM}$ pyrophosphatase in a Quartz SUPRASIL Macro/Semi-micro Cell (Perkin Elmer; catalogue number B0631132) and the initial fluorescence was recorded. NTP substrates $(5 \mu \mathrm{M})$ and RNAP inhibitors were then sequentially added into the cuvette under continuous mixing and incubated for $5 \mathrm{~min}$ before taking each reading. Time-resolved measurements were performed in an Applied Photophysics (Leatherhead, UK) SX.18MV stopped-flow instrument at $25^{\circ} \mathrm{C}$. The reaction was initiated by mixing $60 \mu \mathrm{l}$ of $0.2 \mu \mathrm{M}$ TEC with $60 \mu \mathrm{l}$ of $400 \mu \mathrm{M}$ NTP. Both solutions were prepared in TB10D buffer and, where indicated, supplemented with 0.4-250 $\mu \mathrm{M}$ CBR703. 6-MI fluorophore was excited at $340 \mathrm{~nm}$ and emitted light was collected through $400 \mathrm{~nm}$ longpass filters. At least three individual traces were averaged for each reported curve.

Single-round pause assays. TECs were formed for $15 \mathrm{~min}$ at $37^{\circ} \mathrm{C}$ with $30 \mathrm{nM}$ linear PCR-generated pIA171 template and $40 \mathrm{nM}$ RNAP holoenzyme in $20 \mathrm{mM}$ Tris-acetate, $20 \mathrm{mM}$ Na-acetate, $2 \mathrm{mM} \mathrm{Mg-acetate,} 14 \mathrm{mM}$ 2-mercaptoethanol, $0.1 \mathrm{mM}$ EDTA and $4 \%$ glycerol, $\mathrm{pH}$ 7.9. To halt RNAP after the addition of A29, synthesis was initiated in the absence of UTP, with $150 \mu \mathrm{M}$ ApU, $5 \mu \mathrm{M}$ ATP and GTP, and $1 \mu \mathrm{M}$ CTP supplemented with $\left[\alpha^{-32} \mathrm{P}\right]-\mathrm{CTP}$. For $\beta^{\prime} \mathrm{H} 777 \mathrm{~A}$ enzyme, ATP and GTP were used at $50 \mu \mathrm{M}$ and CTP at $10 \mu \mathrm{M}$ to allow for efficient halted complex formation. Halted complexes were incubated with CBR703 
(or dimethylsulphoxide) for $3 \mathrm{~min}$ at $37^{\circ} \mathrm{C}$. Transcription was restarted by addition of one-tenth volume of $100 \mu \mathrm{M}$ GTP, $1.5 \mathrm{mM}$ CTP, ATP and UTP, and $250 \mathrm{\mu g} \mathrm{ml}^{-1}$ rifapentin. Samples were removed at $7,15,30,45,60,120,180,300$ and $480 \mathrm{~s}$ and after a final $5 \mathrm{~min}$ incubation with $200 \mu \mathrm{M}$ GTP (chase), and were quenched by addition of an equal volume of $10 \mathrm{M}$ urea, $50 \mathrm{mM}$ EDTA, $45 \mathrm{mM}$ Tris-borate; $\mathrm{pH} 8.3,0.1 \%$ bromophenol blue and $0.1 \%$ xylene cyanol. RNAs were separated on $8 \%$ denaturing polyacrylamide gels and quantified using a Typhoon FLA 9000 scanner (GE Healthcare), ImageQuant Software and Microsoft Excel. Each assay was performed in triplicate.

Docking experiments. The three-dimensional structures of CBR703, CBR9379 and CBR9393 were built in Discovery Studio 3.5 (Accelrys, San Diego, CA, USA) and optimized using Minimize Ligands protocol and CHARMM force field ${ }^{46}$. In CBR703 and CBR9379 benzene rings interconnected by $N$-hydroxyamidine, moiety were modelled in cis-configuration, whereas $N$-hydroxyamidine and carbamide (in CBR9379) moieties were modelled planar with non-rotable C-N and C-O bonds. In CBR9393, the bond between the benzene ring and $\alpha$-nitrogen of piperazinylethylamino group was set non-rotable, whereas ternary nitrogen of piperazinyl moiety was protonated and positively charged during docking runs. RNAP fragment comprising amino-acid residues within $20 \AA$ from the putative CBR-binding cavity (Supplementary Data 4) was extracted from E. coli RNAP holoenzyme crystal structure (PDB 4IGC) ${ }^{37}$ and prepared for docking using Prepare Protein protocol of Discovery Studio (for GOLD runs) and AutoDock tools $^{47}$ (for AutoDock Vina runs). GOLD 5.2 (refs 35,36) docking runs were performed using LIGSITE-binding cavity detection algorithm ${ }^{48}$ and GoldScore scoring function. AutoDock Vina 1.1.2 docking runs were performed in $25 \times 25 \times 18 \AA^{3}$ search space centred at $130.9,6.8,-6.7 \AA$ (coordinate space of Supplementary Data 1-4) using default scoring function ${ }^{34}$.

Data analyses. Time-resolved nucleotide incorporation and translocation data were simultaneously fit to a three-step model using the numerical integration capabilities of KinTek Explorer software ${ }^{49}$ (KinTek Corporation). The model postulated that the initial TEC16 slowly and reversibly interconverts between inactive and active states and, on the addition of the NTP substrate, undergoes an irreversible transition to TEC17, followed by irreversible translocation except for $\beta^{\prime}$ F773V and $\beta^{\prime}$ P750L RNAPs where translocation was modelled as a reversible process. Equilibrium titration data were fit to the dissociation equilibrium equations that accounted for changes in concentrations of all reactants on complex formation using Scientist 2.01 software (Micromath, Saint Louis, MO, USA). These models are described in detail in Supplementary Methods.

\section{References}

1. Werner, F. \& Grohmann, D. Evolution of multisubunit RNA polymerases in the three domains of life. Nat. Rev. Microbiol. 9, 85-98 (2011).

2. Svetlov, V. \& Nudler, E. Basic mechanism of transcription by RNA polymerase II. Biochim. Biophys. Acta Gene Regul. Mech. 1829, 20-28 (2013).

3. Ho, M. X., Hudson, B. P., Das, K., Arnold, E. \& Ebright, R. H. Structures of RNA polymerase-antibiotic complexes. Curr. Opin. Struct. Biol. 19, 715-723 (2009).

4. Vassylyev, D. G. et al. Structural basis for substrate loading in bacterial RNA polymerase. Nature 448, 163-168 (2007).

5. Wang, D., Bushnell, D. A., Westover, K. D., Kaplan, C. D. \& Kornberg, R. D. Structural basis of transcription: role of the trigger loop in substrate specificity and catalysis. Cell 127, 941-954 (2006).

6. Malinen, A. M. et al. Active site opening and closure control translocation of multisubunit RNA polymerase. Nucleic Acids Res. 40, 7442-7451 (2012).

7. Ederth, J., Mooney, R. A., Isaksson, L. A. \& Landick, R. Functional interplay between the jaw domain of bacterial RNA polymerase and allele-specific residues in the product RNA-binding pocket. J. Mol. Biol. 356, 1163-1179 (2006).

8. Artsimovitch, I. \& Landick, R. Pausing by bacterial RNA polymerase is mediated by mechanistically distinct classes of signals. Proc. Natl Acad. Sci. USA 97, 7090-7095 (2000).

9. Neuman, K. C., Abbondanzieri, E. A., Landick, R., Gelles, J. \& Block, S. M. Ubiquitous transcriptional pausing is independent of RNA polymerase backtracking. Cell 115, 437-447 (2003).

10. Von Hippel, P. H. \& Pasman, Z. Reaction pathways in transcript elongation. Biophys. Chem. 101-102, 401-423 (2002).

11. Proshkin, S., Rahmouni, A. R., Mironov, A. \& Nudler, E. Cooperation between translating ribosomes and RNA polymerase in transcription elongation. Science 328, 504-508 (2010).

12. Landick, R., Carey, J. \& Yanofsky, C. Translation activates the paused transcription complex and restores transcription of the trp operon leader region. Proc. Natl Acad. Sci. USA 82, 4663-4667 (1985).

13. Roberts, J. W. et al. Antitermination by bacteriophage lambda Q protein. Cold Spring Harb. Symp. Quant. Biol. 63, 319-325 (1998).
14. Artsimovitch, I. \& Landick, R. The transcriptional regulator RfaH stimulates RNA chain synthesis after recruitment to elongation complexes by the exposed nontemplate DNA strand. Cell 109, 193-203 (2002).

15. Nag, A., Narsinh, K. \& Martinson, H. G. The poly(A)-dependent transcriptional pause is mediated by CPSF acting on the body of the polymerase. Nat. Struct. Mol. Biol. 14, 662-669 (2007).

16. Miropolskaya, N., Artsimovitch, I., Klimasauskas, S., Nikiforov, V. \& Kulbachinskiy, A. Allosteric control of catalysis by the F loop of RNA polymerase. Proc. Natl Acad. Sci. USA 106, 18942-18947 (2009).

17. Toulokhonov, I., Zhang, J., Palangat, M. \& Landick, R. A central role of the RNA polymerase trigger loop in active-site rearrangement during transcriptional pausing. Mol. Cell 27, 406-419 (2007).

18. Sydow, J. F. et al. Structural basis of transcription: mismatch-specific fidelity mechanisms and paused RNA polymerase II with frayed RNA. Mol. Cell 34, 710-721 (2009).

19. Sevostyanova, A., Belogurov, G. A., Mooney, R. A., Landick, R. \& Artsimovitch, I. The beta subunit gate loop is required for RNA polymerase modification by RfaH and NusG. Mol. Cell 43, 253-262 (2011).

20. Weixlbaumer, A., Leon, K., Landick, R. \& Darst, S. A. Structural basis of transcriptional pausing in bacteria. Cell 152, 431-441 (2013).

21. Greive, S. J. \& von Hippel, P. H. Thinking quantitatively about transcriptional regulation. Nat. Rev. Mol. Cell Biol. 6, 221-232 (2005).

22. Artsimovitch, I., Chu, C., Lynch, A. S. \& Landick, R. A new class of bacterial RNA polymerase inhibitor affects nucleotide addition. Science 302, 650-654 (2003).

23. Villain-Guillot, P., Gualtieri, M., Bastide, L. \& Leonetti, J. P. In vitro activities of different inhibitors of bacterial transcription against Staphylococcus epidermidis biofilm. Antimicrob. Agents Chemother. 51, 3117-3121 (2007).

24. Svetlov, V., Belogurov, G. A., Shabrova, E., Vassylyev, D. G. \& Artsimovitch, I. Allosteric control of the RNA polymerase by the elongation factor RfaH. Nucleic Acids Res. 35, 5694-5705 (2007).

25. Nedialkov, Y. A. et al. The RNA polymerase bridge helix YFI motif in catalysis, fidelity and translocation. Biochim. Biophys. Acta 1829, 187-98 (2013).

26. Landick, R., Stewart, J. \& Lee, D. N. Amino acid changes in conserved regions of the beta-subunit of Escherichia coli RNA polymerase alter transcription pausing and termination. Genes Dev. 4, 1623-1636 (1990).

27. Jovanovic, M. et al. Activity map of the Escherichia coli RNA polymerase bridge helix. J. Biol. Chem. 286, 14469-14479 (2011).

28. Temiakov, D. et al. Structural basis of transcription inhibition by antibiotic streptolydigin. Mol. Cell 19, 655-666 (2005).

29. Nedialkov, Y. A., Gong, X. Q., Yamaguchi, Y., Handa, H. \& Burton, Z. F. Assay of transient state kinetics of RNA polymerase II elongation. Methods Enzymol. 371, 252-264 (2003).

30. Holmes, S. F., Foster, J. E. \& Erie, D. A. Kinetics of multisubunit RNA polymerases: experimental methods and data analysis. Methods Enzymol. 371, 71-81 (2003).

31. Artsimovitch, I. et al. Tagetitoxin inhibits RNA polymerase through trapping of the trigger loop. J. Biol. Chem. 286, 40395-40400 (2011).

32. Tuske, S. et al. Inhibition of bacterial RNA polymerase by streptolydigin: stabilization of a straight-bridge-helix active-center conformation. Cell 122, 541-552 (2005).

33. Landick, R., Wang, D. \& Chan, C. L. Quantitative analysis of transcriptional pausing by Escherichia coli RNA polymerase: his leader pause site as paradigm. Methods Enzymol. 274, 334-353 (1996).

34. Trott, O. \& Olson, A. J. AutoDock Vina: improving the speed and accuracy of docking with a new scoring function, efficient optimization, and multithreading. J. Comput. Chem. 31, 455-461 (2010).

35. Jones, G., Willett, P. \& Glen, R. C. Molecular recognition of receptor sites using a genetic algorithm with a description of desolvation. J. Mol. Biol. 245, 43-53 (1995).

36. Jones, G., Willett, P., Glen, R. C., Leach, A. R. \& Taylor, R. Development and validation of a genetic algorithm for flexible docking. J. Mol. Biol. 267, 727-748 (1997).

37. Murakami, K. S. X-ray crystal structure of Escherichia coli RNA polymerase б70 holoenzyme. J. Biol. Chem. 288, 9126-9134 (2013).

38. Herbert, K. M. et al. Sequence-resolved detection of pausing by single RNA polymerase molecules. Cell 125, 1083-1094 (2006).

39. Weinzierl, R. O. The nucleotide addition cycle of RNA polymerase is controlled by two molecular hinges in the Bridge Helix domain. BMC Biol. 8, 134 (2010).

40. Hein, P. P. \& Landick, R. The bridge helix coordinates movements of modules in RNA polymerase. BMC Biol. 8, 141 (2010).

41. Brueckner, F. \& Cramer, P. Structural basis of transcription inhibition by alphaamanitin and implications for RNA polymerase II translocation. Nat. Struct. Mol. Biol. 15, 811-818 (2008).

42. Heikinheimo, P. et al. A site-directed mutagenesis study of Saccharomyces cerevisiae pyrophosphatase. Functional conservation of the active site of soluble inorganic pyrophosphatases. Eur. J. Biochem. 239, 138-143 (1996). 
43. Belogurov, G. A. et al. Structural basis for converting a general transcription factor into an operon-specific virulence regulator. Mol. Cell 26, 117-129 (2007).

44. Komissarova, N., Kireeva, M. L., Becker, J., Sidorenkov, I. \& Kashlev, M. Engineering of elongation complexes of bacterial and yeast RNA polymerases. Methods Enzymol. 371, 233-251 (2003).

45. Abramoff, M. D., Magalhaes, P. J. \& Ram, S. J. Image processing with ImageJ. Biophotonics Int. 11, 36-42 (2004).

46. Brooks, B. R. et al. CHARMM: a program for macromolecular energy, minimization, and dynamics calculations. J. Comput. Chem. 4, 187-217 (1983).

47. Sanner, M. F. Python: a programming language for software integration and development. J. Mol. Graph. Model. 17, 57-61 (1999).

48. Hendlich, M., Rippmann, F. \& Barnickel, G. LIGSITE: automatic and efficient detection of potential small molecule-binding sites in proteins. J. Mol. Graph. Model. 15, 389 (1997).

49. Johnson, K. A. Fitting enzyme kinetic data with KinTek Global Kinetic Explorer. Methods Enzymol. 467, 601-626 (2009).

50. Bae, B. et al. Phage T7 Gp2 inhibition of Escherichia coli RNA polymerase involves misappropriation of $\sigma 70$ domain 1.1. Proc. Natl Acad. Sci. USA 110, 19772-19777 (2013).

51. Zuo, Y., Wang, Y. \& Steitz, T. A. The mechanism of E. coli RNA polymerase regulation by ppGpp is suggested by the structure of their complex. Mol. Cell 50, 430-436 (2013).

52. Opalka, N. et al. Complete structural model of Escherichia coli RNA polymerase from a hybrid approach. PLoS Biol. 8, e1000483 (2010).

53. Santangelo, T. J. \& Artsimovitch, I. Termination and antitermination: RNA polymerase runs a stop sign. Nat. Rev. Microbiol. 9, 319-329 (2011).

54. Toulokhonov, I., Artsimovitch, I. \& Landick, R. Allosteric control of RNA polymerase by a site that contacts nascent RNA hairpins. Science 292, 730-733 (2001).

55. Ha, K. S., Toulokhonov, I., Vassylyev, D. G. \& Landick, R. The NusA $\mathrm{N}$-terminal domain is necessary and sufficient for enhancement of transcriptional pausing via interaction with the RNA exit channel of RNA polymerase. J. Mol. Biol. 401, 708-725 (2010).

56. Bochkareva, A., Yuzenkova, Y., Tadigotla, V. R. \& Zenkin, N. Factorindependent transcription pausing caused by recognition of the RNA-DNA hybrid sequence. EMBO J. 31, 630-639 (2012).

57. Vassylyev, D. G., Vassylyeva, M. N., Perederina, A., Tahirov, T. H. \& Artsimovitch, I. Structural basis for transcription elongation by bacterial RNA polymerase. Nature 448, 157-162 (2007).

58. Zhang, Y. et al. Structural basis of transcription initiation. Science 338, 1076-1080 (2012).

59. Campbell, E. A. et al. Structural mechanism for rifampicin inhibition of bacterial RNA polymerase. Cell 104, 901-912 (2001).

60. Artsimovitch, I. et al. Allosteric modulation of the RNA polymerase catalytic reaction is an essential component of transcription control by rifamycins. Cell 122, 351-363 (2005).
61. Mukhopadhyay, J. et al. The RNA polymerase "switch region" is a target for inhibitors. Cell 135, 295-307 (2008).

62. Srivastava, A. et al. New target for inhibition of bacterial RNA polymerase: 'switch region'. Curr. Opin. Microbiol. 14, 532-543 (2011).

63. Chakraborty, A. et al. Opening and closing of the bacterial RNA polymerase clamp. Science 337, 591-595 (2012).

64. Belogurov, G. A. et al. Transcription inactivation through local refolding of the RNA polymerase structure. Nature 457, 332-335 (2009).

\section{Acknowledgements}

This work was supported by the Academy of Finland Grants 130581 and 263713 to G.A.B. and NIH Grant GM067153 to I.A. Salary for M.T. was paid by the National Doctoral Program in Informational and Structural Biology. We acknowledge CSC-IT Center for Science for providing national licence to the Discovery Studio program and computational resources used for ligand docking with GOLD. Essential equipment was contributed by Walter and Lisi Wahl Foundation.

\section{Author contributions}

A.M.M., M.T. and H.M. performed nucleotide addition and translocation measurements. M.NM. performed pausing assays under the guidance of I.A.; A.M.M. and G.A.B. constructed CBR-RNAP models. A.M.M. and T.G. constructed vectors expressing mutationally altered RNAPs and purified RNAP enzymes. G.A.B. supervised the study and interpreted the results. G.A.B. and I.A. wrote the manuscript.

\section{Additional information}

Supplementary Information accompanies this paper at http://www.nature.com/ naturecommunications

Competing financial interests: The authors declare no competing financial interests.

Reprints and permission information is available online at http://npg.nature.com/ reprintsandpermissions/

How to cite this article: Malinen, A. M. et al. CBR antimicrobials alter coupling between the bridge helix and the $\beta$ subunit in RNA polymerase. Nat. Commun. 5:3408 doi: $10.1038 /$ ncomms4408 (2014)

(c) (1) (2) This work is licensed under a Creative Commons AttributionDonCommercial-ShareAlike 3.0 Unported License. The images or other third party material in this article are included in the article's Creative Commons license, unless indicated otherwise in the credit line; if the material is not included under the Creative Commons license, users will need to obtain permission from the license holder to reproduce the material. To view a copy of this license, visit http:// creativecommons.org/licenses/by-nc-sa/3.0/ 